A. Pramesh Rao, G. Swarup and Gopal-Krishna, eds.

\title{
WSRT 1.4 GHz Observations of the Hubble Deep Field
}

\author{
M.A. Garrett \\ JIVE, Postbus 2, 7990 AA, Dwingeloo, NL \\ G. de Bruyn, W. Baan \\ NFRA, Postbus 2, 7990 AA, Dwingeloo, NL \\ R.T. Schilizzi \\ JIVE, Postbus 2, 7990 AA, Dwingeloo, NL
}

\begin{abstract}
We present WSRT $1.38 \mathrm{GHz}$ observations of the Hubble Deep Field (and flanking fields). 72 hours of data were combined to produce the WSRT's deepest image yet, reaching an r.m.s. noise level of 8 microJy/beam. We detect radio emission from galaxies both in the HDF and HFF which have not been previously detected by recent MERLIN or VLA studies of the field.
\end{abstract}

\section{Background, Observations and Preliminary Results}

Deep Radio observations of the Hubble Deep Field region are now advancing our understanding of the faint microJy radio source population. In particular, VLA and MERLIN observations of the HDF (Richards et al. 1999, Muxlow et al. 1999) suggest that faint sub-mJy and microJy radio sources are mostly identified with star forming galaxies, often located at moderate to high redshifts.

In the period April-May 1999 we observed the HDF and HFF with the newly upgraded Westerbork Synthesis Radio Telescope (WSRT) at $1.4 \mathrm{GHz}$ for a total of 72 hours. Our aim was to utilise the WSRT's superb brightess sensitivity to extend the investigation of the microJy source population to extended radio sources that might otherwise be resolved out or go undetected in the previous higher resolution or higher frequency radio observations.

Fig. 1 shows the WSRT image of the HDF/HFF convolved with a circular 15 arcsecond Gaussian restoring beam. This represents the deepest image made with the WSRT to date, reaching a rms noise level of $8 \mu \mathrm{Jy} / \mathrm{beam}$. We detect radio emission from galaxies both in the HDF and HFF which have not been previously detected by recent MERLIN or VLA studies of the field. More than 30 new $(>5 \sigma)$ detections have been obtained in a $10 \times 10$ arcmin field, centred on the HDF. Some of these sources are actually blends of two or more sources but a large percentage are also discrete. Three of the new $1.4 \mathrm{GHz}$ sources are located in the HDF itself, and have infra-red ISO detections. Two of the three are associated with Spiral galaxies and the third is an irregular galaxy (the latter is detected by the VLA at 8.4 but not $1.4 \mathrm{GHz}$ ). The new WSRT detections indicate that 
perhaps a significant fraction of starburst galaxies present more extended radio emission than the previous (higher resolution) VLA and MERLIN observations suggest.

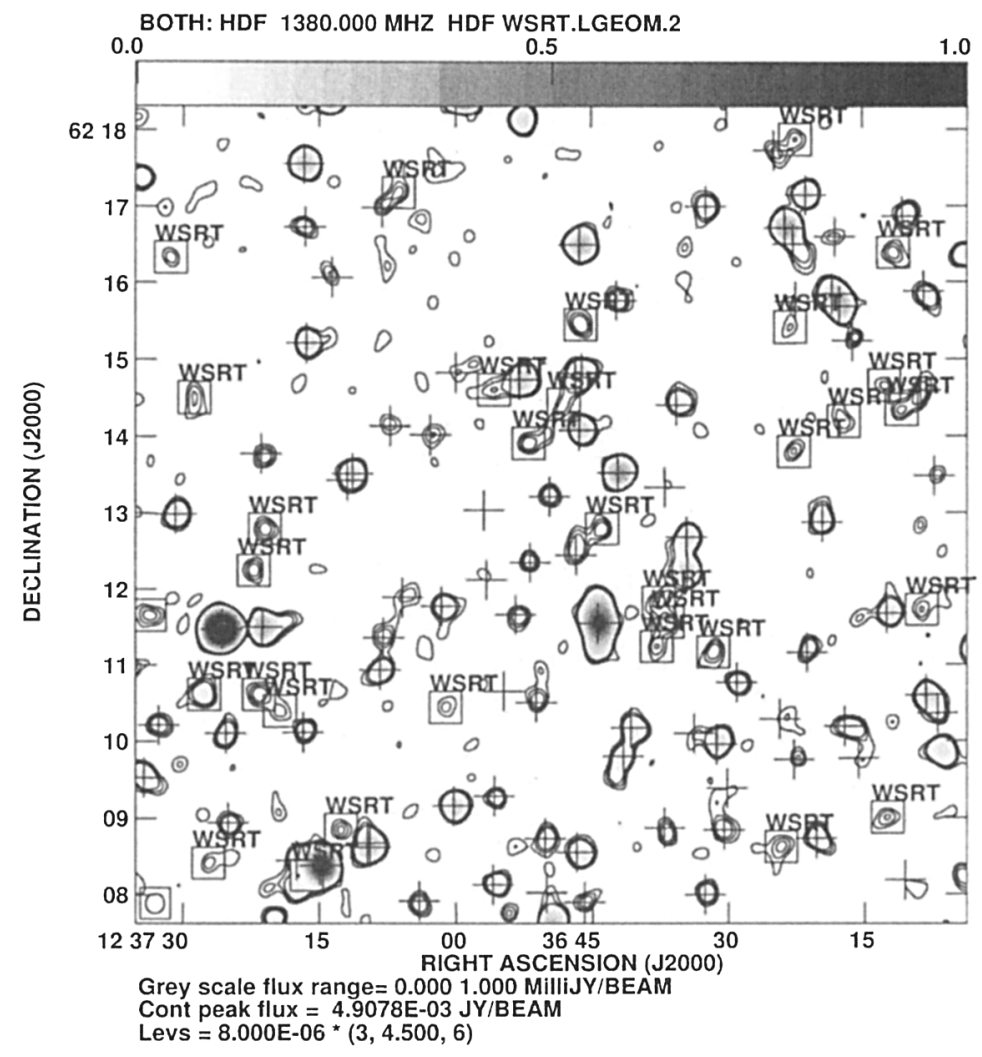

Figure 1. A $10 \times 10$ arcmin image of the HDF and flanking Fields. Sources previously detected by the VLA are indicated with a cross. Additional sources detected by the WSRT are indicated with a box and label

\section{References}

Muxlow, T.W.B., Wilkinson, P.N., Richards, A.M.S., Kellerman, K.I., Richards, E.A., Garrett, M.A. (1999) New Astronomy Reviews, 43, 623.

Richards, E. A., Kellermann, K. I., Fomalont, E. B., Windhorst, R. A., Partridge, R. B. (1998) $A J$, 116, 1039. 